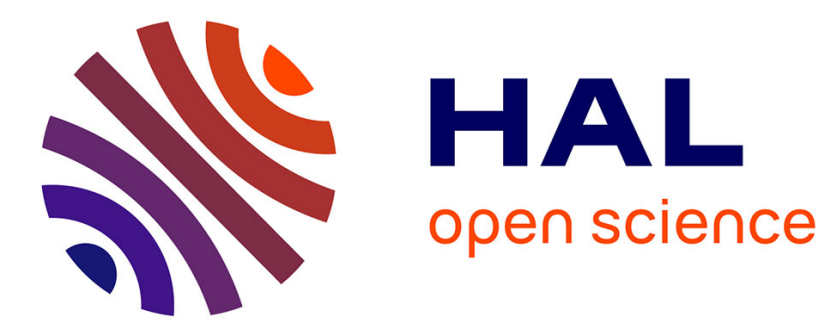

\title{
Dépasser la crise de l'histoire de l'intégration européenne
}

Laurent Warlouzet

\section{To cite this version:}

Laurent Warlouzet. Dépasser la crise de l'histoire de l'intégration européenne. Politique européenne, 2014, 44 (2), pp.98. 10.3917/poeu.044.0098 . hal-02560516

\section{HAL Id: hal-02560516 https://hal.science/hal-02560516}

Submitted on 13 May 2020

HAL is a multi-disciplinary open access archive for the deposit and dissemination of scientific research documents, whether they are published or not. The documents may come from teaching and research institutions in France or abroad, or from public or private research centers.
L'archive ouverte pluridisciplinaire HAL, est destinée au dépôt et à la diffusion de documents scientifiques de niveau recherche, publiés ou non, émanant des établissements d'enseignement et de recherche français ou étrangers, des laboratoires publics ou privés. 


\title{
DÉPASSER LA CRISE DE L'HISTOIRE DE L'INTÉGRATION EUROPÉENNE
}

\author{
Laurent Warlouzet
}

L'Harmattan | Politique européenne

\author{
$2014 / 2-n^{\circ} 44$ \\ pages 98 à 122
}

ISSN 1623-6297

Article disponible en ligne à l'adresse:

http://www.cairn.info/revue-politique-europeenne-2014-2-page-98.htm

Pour citer cet article :

Warlouzet Laurent, « Dépasser la crise de l'histoire de l'intégration européenne »,

Politique européenne, 2014/2 n44, p. 98-122.

Distribution électronique Cairn.info pour L'Harmattan.

(C) L'Harmattan. Tous droits réservés pour tous pays.

La reproduction ou représentation de cet article, notamment par photocopie, n'est autorisée que dans les limites des conditions générales d'utilisation du site ou, le cas échéant, des conditions générales de la licence souscrite par votre établissement. Toute autre reproduction ou représentation, en tout ou partie, sous quelque forme et de quelque manière que ce soit, est interdite sauf accord préalable et écrit de l'éditeur, en dehors des cas prévus par la législation en vigueur en France. II est précisé que son stockage dans une base de données est également interdit. 


\section{POLITIQUE EUROPÉENNE \\ $\mathbf{N}^{\circ} 44 \mid 2014$}

Laurent Warlouzet

[p. 98-122]

\section{Dépasser la crise de l’histoire de l’intégration européenne}

Les historiens de l'intégration européenne utilisent abondamment la notion de « crise », associée à celle de "relance » pour décrire les différentes étapes de l'évolution des Communautés européennes. Cet usage traduit souvent un centrage implicite sur l'Europe des Six, répondant en outre à une certaine tentation téléologique. Cette dérive, qui a largement été dénoncée par les contempteurs de l'histoire de l'intégration européenne, s'accompagne d'une situation de crise du domaine d'étude en tant que tel. Cependant, l'histoire de l'intégration européenne a aujourd'hui largement renouvelé ses méthodes et ses angles d'approche, permettant d'espérer une « relance » autour de la notion d'« histoire des coopérations européennes ».

\section{European Integration History: Beyond the Crisis}

Widely used by historians of European integration, the notion of "crisis" has frequently been associated with "relaunching" the Community. This use betrays an implicit focus on the 6 founding members as well as a teleological temptation. As a result, the field of European integration history has been accused by its critics of being itself in crisis. Nowadays however, the methods and conceptual approaches of European integration history are being renewed through the "history of European cooperation." 


\title{
Dépasser la crise de l'histoire de l'intégration européenne
}

\author{
Laurent Warlouzet \\ London School of Economics and Political Science
}

a notion de crise est omniprésente dans l'histoire de l'intégration européenne car elle repose sur un projet ambitieux: dépasser l'échelle des États-nations au moment même où leurs prérogatives se sont considérablement accrues avec les guerres totales et le développement des États-providence dans les deux premiers tiers du $\mathrm{xx}^{\mathrm{e}}$ siècle. L’idée européenne a été promue au sommet par différents responsables politiques depuis 1919, puis a connu une mise en œuvre heurtée à partir de 1948 (Guieu et al., 2007). De nombreux projets échouèrent, tandis que l'organisation la plus importante sur le plan institutionnel, l'Union européenne (UE), subit aujourd'hui de nombreuses critiques, en particulier depuis la crise de la zone euro.

Le terme de « crise » est donc étroitement associé à l’histoire de l'intégration européenne. Provenant du mot grec Krisis signifiant « décision », la « crise » désigne dans le domaine médical un tournant: le moment où une maladie connaît un changement subit et décisif, en bien ou en mal. En chinois, le mot « crise » est formé de deux caractères, l'un signifiant « danger » et l'autre « opportunité ». Ainsi, la notion de crise est associée soit à un échec définitif, soit à une relance du processus de coopération en Europe. Elle est particulièrement attachée à l'histoire de l'« intégration » européenne au sens strict, celle des communautés européennes, depuis la Communauté économique du charbon et de l'acier (CECA) de 1951, en passant par la Communauté économique européenne (CEE) de 1957 jusqu’à l'actuelle Union européenne (UE) née en 1992.

Bien plus, comme toute histoire du temps présent, l'histoire de l'intégration européenne est influencée par l'actualité. En l'occurrence, la défiance dont souffrent les communautés européennes chez une part croissante de la population depuis la fin du $\mathrm{xx}^{\mathrm{e}}$ siècle n'épargne pas ses historiens, amenant certains à s'interroger sur une crise de la discipline elle-même. Il s'agit donc d'étudier la notion de crise dans sa multiplicité, en se concentrant sur l'interaction entre la crise de l'objet d'étude et celle de la communauté scientifique. Après 
avoir montré que la scansion des « crises » et des « relances » fait aujourd'hui l'objet d'une interprétation renouvelée par rapport à la vision classique (I), nous reviendrons sur les origines du constat de crise de la discipline (II). Nous verrons alors que cette approche pessimiste doit aujourd'hui être dépassée pour opérer une véritable « relance » de ce domaine d'étude vers une « histoire des coopérations européennes » (III).

\section{La scansion des « crises " et des « relances "}

L'alternance des « crises » et des « relances » est au cour d'une vision de l'histoire de l'intégration européenne qui peut être qualifiée de « classique », même si cette analyse est aujourd’hui largement renouvelée.

\section{La vision classique}

La vision classique de l'histoire de l'intégration européenne décrite dans les manuels repose sur une succession de «crises » et de « relances » dans un mouvement linéaire de construction d'une Europe communautaire. Cette dynamique débute avec la déclaration Schuman du 9 mai 1950 annonçant la Communauté européenne du charbon et de l'acier (CECA). De la CECA, un lien est ensuite tissé avec la Communauté économique européenne (CEE) de 1957 puis avec l'Union européenne (UE) de 1992. L'adoption du 9 mai 1950 comme date fondatrice du processus de construction de l'Europe communautaire permet, selon Antonin Cohen, de gommer les échecs des premières années de l'intégration européenne tout en traçant un continuum entre CECA et CEE (Cohen, 2007, 19). Elle a été notamment promue par les laudateurs de Jean Monnet, l'auteur principal de la déclaration Schuman.

À partir du 9 mai 1950 se développe un récit héroïque, où les promoteurs de l'idée européenne représentent en général le progrès, face à des logiques nationales considérées comme rétrogrades. C'est particulièrement le cas dans l'ouvrage au titre évocateur, L'Europe difficile de Bino Olivi, souvent cité comme un manuel de référence (Olivi et Giacone, 2007). De fait, l'ouvrage est une bonne synthèse mais il adopte sans ambiguïté le point de vue des institutions communautaires. Bino Olivi n'est d'ailleurs pas un historien mais un ancien fonctionnaire européen qui exerçait la fonction de porte-parole de la Commission européenne. 
À l'euphorie de la création de la CECA, complétée par la Communauté européenne de défense (CED) conclue en 1952 et à la Communauté politique européenne projetée en 1953, succède une première « crise ». Il s'agit du rejet de la CED par le Parlement français le 30 août 1954. Déjà dans la Querelle de la $C E D$, Raymond Aron reliait l'ambitieux projet d'armée européenne à une volonté de relance de l'Europe fédérale, brisée par cette crise (Aron, 1956). Elle est suivie d'une première « relance » à Messine, en juin 1955, lorsque les ministres des Affaires étrangères des six pays membres de la CECA décident de lancer un processus qui aboutit en mars 1957 à la signature des traités de Rome créant la CEE et l'Euratom. La « crise » concerne alors la Grande-Bretagne, qui «manque le train » de Messine («Missed the boat» selon Anne Deighton, 1990) et ne parvient pas à promouvoir son projet alternatif de Zone de libre-échange.

Du côté de la CEE, les premières années sont une véritable « lune de miel » entre les États membres et la Commission européenne, selon l'expression de l'influente autobiographie du premier vice-président français de la Commission européenne Robert Marjolin (1986, 304). Ensuite s’ouvre « le temps des crises » à partir de 1963 (Ibid., 1986, 322). Il s'agit des deux rejets unilatéraux de la Grande-Bretagne par le président français Charles de Gaulle (1963 et 1967) et de la « crise de la Chaise vide » (1965-1966). La marche inexorable vers une communauté européenne toujours plus supranationale et toujours plus large est interrompue. Il faut la « relance » du sommet européen de La Haye en décembre 1969 pour débloquer les dossiers en suspens depuis 1963-1965, soit l'adhésion britannique et le financement de la Politique agricole commune (PAC). Le triptyque « achèvement/approfondissement élargissement » est alors officiellement proclamé, et est repris ensuite par les historiens, comme en témoigne le titre d'un important colloque portant sur cette période qui reprend ce triptyque (Van der Harst, 2007).

Les années 1970 cadrent mal avec la dialectique « crise » et « relance » car elles sont caractérisées par une série de réformes institutionnelles successives (traité de 1970, création du Conseil européen, élection du Parlement européen au suffrage universel, arrêts de la Cour renforçant le droit européen) qui ne se cristallisent pas tous dans un moment précis de relance, mais s'étalent au contraire tout au long de la période. De même, les problèmes suscités par la Grande-Bretagne entraînent des crises s'égrenant sur une décennie. La renégociation de 1975 porte les germes d'une crise potentielle, évitée in fine, avant que la question budgétaire ne dégénère en crise ouverte en 1982- 
1984. C'est la British Budgetary Question (BBQ), ou Bloody British Question ${ }^{1}$ (Ludlow, 2007). Là encore, la crise est dépassée par une troisième relance, celle du sommet de Fontainebleau en juin 1984. Les bases de l'activisme de la Commission Delors sont alors posées. La ratification difficile du traité de Maastricht en 1992-1993 ouvre une nouvelle période de crise, ouverte ou latente, dont l'Union européenne a du mal à sortir. Ainsi se succèdent des crises plus ou moins longues, et des relances ponctuelles, qui portent le nom de la ville accueillant le sommet de chefs d'État et de gouvernement décisif.

Cette vision d'une alternance entre crises et relances est centrée sur l'ensemble CECA-CEE-UE. Les autres formes de coopération européenne ne sont pas ignorées mais sont délaissées en tant que représentantes imparfaites de l'idéal européen. Pourtant, l'Organisation européenne de coopération économique (OECE), créée en 1948, tout comme le Conseil de l'Europe, né en 1949, sont antérieurs à la CECA dont le traité fondateur date de 1951. Par ailleurs, l'accent est mis sur les « grands hommes » (ou femmes comme Margaret Thatcher), qui s'affrontent dans des combats homériques. Le détail des négociations est souvent survolé pour mettre en valeur des « gagnants » et des « perdants ». En ce sens, le récit classique de l'histoire de l'intégration européenne rejoint celui des histoires nationales idéalisées.

\section{Une analyse renouvelée}

Ce récit classique est toutefois moins celui des historiens spécialistes de l'intégration européenne, que des auteurs de manuels ou de mémoires. Le recours aux archives permet aux historiens non pas de contester ce schéma général crise/relance, qui conserve sa validité, mais d'en approfondir les ressorts, en dénonçant les simplifications abusives de l'histoire des grands hommes.

Ainsi, la perspective traditionnelle est revisitée dans le cas britannique où la thèse du «missing the boat » a été battue en brèche par les études sur archives menées par Wolfram Kaiser et Alan Milward. Milward souligna la 
rationalité de la politique britannique, dont une partie importante des relais d'influence se situaient alors à une échelle mondiale (Milward, 2002, 2-3). De son côté, Kaiser attribue la décision britannique de rester en dehors de la CEE à son " great power syndrome » (Kaiser, 1996, 56) et à son insertion dans un espace culturel nord-européen dont les valeurs sont sensiblement différentes de l'Europe carolingienne qui constituait le cœur de l'Europe des Six (Kaiser, 2001). Ainsi, si la méthode employée dans les deux monographies reste classique - le recours aux archives officielles d'un seul pays -, les conclusions sont nouvelles. Surtout W. Kaiser introduit une perspective constructiviste qui renouvelle les problématiques, lorsqu'il identifie un rapport aux coopérations européennes propre aux pays d'Europe du Nord.

Plus stimulant encore sont les ouvrages fondés sur les archives des institutions européennes d'un ou de plusieurs pays. L'exemple magistral est le livre de Piers Ludlow intitulé The European Community and the Crises of the 1960s (Ludlow, 2006). La notion de crise est au cœur de cette étude qui se concentre sur la période 1963-1969. Le rejet de la candidature britannique par le général de Gaulle en janvier 1963 ouvre l'étude. Comme il l'avait démontré dans sa thèse (Ludlow, 1997, 236-237), P. Ludlow confirme que la France n'était pas le seul État méfiant envers la Grande-Bretagne. L'Italie et la RFA nourrissaient également de sérieuses inquiétudes face à un partenaire britannique intransigeant et exigeant une renégociation des accords anciens. La même dynamique est à l'œuvre durant la crise de la Chaise vide, où $P$. Ludlow souligne l'hypocrisie des discours officiels. La France gaullienne est plus attachée à la CEE qu'elle ne le dit, et doit se contenter d'une demi-victoire (ou d'une demi-défaite) avec le compromis de Luxembourg ${ }^{2}$, pourtant considéré comme un éclatant succès dans l'historiographie gaulliste. Le président français aurait voulu une renégociation du traité, mais il a dû se concentrer sur une déclaration interprétative unilatérale relative à la préservation du droit de veto ${ }^{3}$. Inversement, beaucoup de décideurs dans les gouvernements des cinq autres États de la CEE se montrent moins empressés de défendre la Commission qu'ils ne le prétendent. Le chancelier allemand Erhard par exemple n'était pas favorable aux ambitions fédéralistes du président Hallstein, de Luxembourg » du 30 janvier 1966, l'usage du vote à la majorité qualifiée, prévu par le traité de Rome, est réduit au profit d'un recours au veto dans certaines circonstances (Warlouzet, 2011a, 253). estime que, lorsqu'il s'agit d'intérêts très importants, la discussion devra se poursuivre jusqu'à ce que l'on soit parvenu à un accord unanime. » 
et était satisfait de conserver un droit de veto que la RFA avait largement utilisé sur la PAC. L'histoire classique de la construction européenne est ainsi démystifiée par l'exposition du décalage entre le discours officiel et la réalité des négociations derrière les portes closes.

La même approche a été utilisée par Ann-Christian Knudsen à propos des négociations sur la PAC, où elle a démontré que la RFA était bien un facteur de crise, à rebours de l'image idéalisée du bon élève (Knudsen, 2006). Kiran Patel a confirmé cette vision de la RFA comme « Veto Player $n^{\circ} 1$ » entre 1959 et 1964 (Patel, 2009). L'unité de l'acteur étatique a ainsi été démontée par l'étude des divisions internes, par exemple entre un ministère de l'Agriculture allemand intéressé par la PAC et défenseur de prix très élevés, et les autres ministères, notamment les Finances, fondamentalement hostiles à cette logique inflationniste. D’autres études ont participé de cette tendance d'une déconstruction de l'acteur étatique national, explicitant tant les divisions internes que les liens d'interdépendance complexe tissés avec la nouvelle administration communautaire. Ainsi, dans le cas français, les commissaires et hauts-fonctionnaires français en poste à Bruxelles ont pu développer une vision économique volontariste, certes fondées sur des références nationales, mais distincte de celle du gouvernement de Paris (Warlouzet, 2011a, 433-444). Dans ce cas, les crises des années 1963-1967 ont servi de détonateur pour l'émergence à Paris et à Bruxelles (chez certains Français en poste au sein de la Commission européenne) d'un groupe « révisionniste » contestant les orientations traditionnelles de la politique européenne française, en particulier la priorité absolue dévolue à la PAC et le rejet a priori de la candidature de la Grande-Bretagne. En matière monétaire, l'image d'Épinal d'une crise profonde des ambitions européennes en la matière entre le choc pétrolier de 1973 et la « relance » de cette thématique monétaire par le discours Jenkins de 1977 a été battue en brèche par une étude récente (Mourlon-Druol, 2012). Cette dernière a mis en valeur la persistance des réflexions dans ce domaine, notamment au sein d'un réseau transnational d'experts, et la continuité des schémas institutionnels entre le «Serpent » de 1972 et le Système monétaire européen (SME) de 1978. Plus généralement, l'étude par le prisme des politiques publiques permet de reconsidérer la chronologie habituelle d'un développement continu car chaque domaine a son rythme propre. Ainsi, la politique sociale a connu un développement assez net pendant les années 1950, au sein de la CECA, et en lien avec les débats internationaux dans le cadre de l'Organisation internationale du travail (OIT) (Mechi, 2012, 160161). L'épisode crise de la CED/relance de Messine ne l'a pas affecté car la politique sociale n'a pas profité de la CEE, où les commissaires italiens en 
charge de ce dossier étaient peu efficaces dans les années 1960. Par contre, elle a bénéficié de la « relance de La Haye » de 1969 grâce en particulier au volontarisme du chancelier Brandt dans ce domaine (Mechi, 2006, 111-115).

L’intensité des crises n’a donc pas été niée par ce renouvellement historiographique fondé sur l'exploitation d'archives, mais leur dimension héroïque d'affrontement de grands hommes, ou d'États monolithiques, a été dépassée. Les oppositions d'acteurs ont été réinsérées dans une double tension, à l'échelle nationale entre des intérêts nationaux composites d'une part, et à l'échelle communautaire du fait de l'interaction complexe entre appareils étatiques nationaux et communautaires d'autre part. En dépit de ce renouvellement, l'histoire de l'intégration européenne reste critiquée pour son approche trop traditionnelle, dont la concentration sur le couple crise/relance participe.

\section{La perception d'une crise de l'histoire de l'intégration européenne}

L'histoire de l'intégration européenne est souvent décrite aujourd'hui comme une discipline en crise. Si ce constat s'explique notamment par le poids important des non-historiens dans l'animation de certains débats majeurs, les difficultés spécifiques de cette histoire doivent être identifiées.

\section{Le constat de crise}

La « crise » de l'histoire de l'intégration européenne est un constat largement partagé au sein des acteurs mêmes de cette histoire. Cette vision a été diffusée par un article influent de l'historien Mark Gilbert (2008), et dans des bilans historiographiques dressés par Wolfram Kaiser et Piers Ludlow (Kaiser 2005, 2008, 2010; Ludlow, 2010). Ces deux historiens sont d'éminents représentants du renouvellement de la discipline. Ils se livrent à une analyse sévère. Elle part du constat d'une large ignorance des historiens de la construction européenne dans les histoires générales de l'Europe au $\mathrm{xx}^{\mathrm{e}}$ siècle qui servent de références dans le monde anglo-saxon, comme celle d'Eric Hobsbawm, d'Harold James, de Tony Judt ou de Mark Mazower (Gilbert, 2008, 655; Kaiser, 2010, 47-48). On pourrait même étendre ce constat aux nombreux politistes recourant à l'approche historique, qui oublient de citer 
les historiens en dehors de l'habituelle référence à A. Milward, seul rescapé de l'isolement de la chapelle des historiens de l'intégration européenne.

Les explications de cet isolement sont triples. Sur le plan méthodologique tout d'abord, W. Kaiser déplore le sous-développement conceptuel de l'histoire de l'intégration européenne et son caractère trop descriptif (Kaiser, 2010, 49). Lui-même auteur d'une thèse fondée sur les méthodes de l'histoire diplomatique, W. Kaiser en dénonce certains excès lorsqu'il néglige les divisions internes aux États (Kaiser, 2005, 208). Plus généralement, il s'agit de ne pas réifier l'État comme acteur monolithique selon l'expression de Laurence Badel et d'Hélène Michel (2011, 15). En termes de sujet ensuite, le domaine est décrié comme trop autocentré. Concentré sur l'histoire de la CEE, il négligerait les autres développements européens (OCDE, etc.), tout comme les rapports avec les autres parties du monde (Patel, 2013). Enfin et surtout, l'approche est dénoncée comme trop téléologique, c'est-à-dire envisageant le passé en fonction d'une fin, qui peut être le présent d'une Union européenne dotée de larges prérogatives, ou d'un futur idéalisé, en particulier celui de l'Europe fédérale qui a animé certains pionniers de la discipline comme l’Allemand Walter Lipgens.

La critique de la téléologie a d'abord été discutée par A. Milward dans son ouvrage The Rescue of the Nation-State qui s'est imposé comme un standard. Il y remet en cause notamment la méthodologie employée par Lipgens, qui a compilé les déclarations pro-européennes de nombreux acteurs des années 1940 sans tenir compte ni du contexte ni de l'influence limitée que ces derniers ont pu avoir sur le processus de décision (Milward, 1992, 15-16). Enfin, dans un chapitre intitulé "The lives and teaching of the European saints », il déconstruit le mythe des « Pères de l'Europe » en expliquant que ces acteurs, au premier rang desquels Jean Monnet, ont soutenu la construction européenne car elle coïncidait avec l'intérêt matériel de leur pays, et pas par idéalisme (Milward, 1992, 319-337). Mark Gilbert a repris cette critique en l'affinant. Il dénonce le fait qu'une partie de l'historiographie est trop centrée sur la CEE, et même sur l'Europe des Six. Il dénonce un présupposé téléologique visible dans l'utilisation d'expression comme « avancées » ou «progrès », tandis que les moments de crise sont souvent dénoncés comme «bloquant » ou " obstruant » un processus d'intégration européenne perçu comme inéluctable (Gilbert, 2008, 645-647). Ce bilan est aggravé par le rôle parfois secondaire joué par les historiens au sens strict dans les débats scientifiques. 


\section{Une place importante des non-historiens}

Ironiquement, le livre le plus cité dans le domaine de l'histoire de l'intégration européenne, si l'on se limite aux auteurs encore actifs, a été écrit par un politiste, en l'occurrence Andrew Moravcsik. Son célèbre The Choice for Europe décrit la période 1955-1992 d'une manière certes univoque, mais en même temps documentée (Moravcsik, 1998). Si l'auteur n'a pas consulté d'archives et a été largement critiqué pour son utilisation des sources et sa volonté de vouloir faire entrer à toute force les faits dans sa théorie de l'intergouvernementalisme libéral (Lieshout, Segers, et van der Vleuten, 2004), son livre reste un tour de force. Il a utilisé une littérature en plusieurs langues et fait preuve de capacités de synthèse et d'analyse remarquables. Son interprétation globale a été souvent contestée mais elle reste puissante. L'ouvrage n'a d'ailleurs pas suscité de réponse véritable de la communauté historienne, incapable de produire des ouvrages interprétatifs couvrant à la fois plusieurs décennies et des domaines larges. Les seules réponses existantes sont des études de cas sur les années 1960 et 1970. La seule tentative d'envergure émane de John Gillingham (2003) dans un ouvrage qui fait figure de contre-exemple sur le plan méthodologique, tant il relève du pamphlet. Il est considéré comme «à peu près aussi fiable sur l'intégration européenne que l'est le Da Vinci de Dan Brown sur le développement historique de l'église catholique » (Ludlow, 2010, 53).

Bien plus, les grands débats sur l'interprétation de l'intégration européenne sont souvent animés par des politistes dans des ouvrages à forte connotation historique (Parsons, 2003; Rittberger, 2005; Vauchez, 2013). Même les juristes développent des études historiques fondées sur archives à propos de thèmes extrêmement précis, tels que l'interprétation sur le long terme d'articles du traité de Rome afin de prendre position dans des débats contemporains sur la modernisation de la politique de la concurrence (Akman, 2009). Cet investissement des juristes dans le domaine de l'intégration européenne avait d'ailleurs suscité l'étonnement ironique d'A. Milward dès 1975: « For lawyers Community law early became an essential area of study, surely the first time lawyers ever led an innovatory movement in education. " (Milward, 1975, 73). La vision historique et juridique a donné lieu au paradigme de l'« integrationthrough law », dont la discussion constitue l'un des champs actuels de débat sur l'intégration européenne. Investi depuis peu par des historiens (Davies et Rasmussen, 2012), un tel champ de recherche reste largement dominé 
par des politistes, sociologues et juristes utilisant la méthode historique ${ }^{4}$. Un ouvrage récent du juriste Peter Lindseth (qui possède il est vrai un doctorat en histoire) propose d'ailleurs une interprétation plus intergouvernementale de ces développements, en s'appuyant notamment sur Milward et sur Moravcsik (Lindseth, 2010, 41-2, 118, 149). Cette approche par le droit permet de relativiser le couple crise/relance en introduisant une nouvelle chronologie. La coloration dramatique de la crise de la Chaise vide de 1965 est patinée d'une couleur nouvelle si les arrêts fondateurs du droit communautaire de 1963-1964 sont pris en compte. La première dynamique est compensée par la seconde. Les années 1970, largement oubliées entre la relance de La Haye de 1969 et la crise budgétaire de 1982-1983, sont également revalorisées sous cette perspective, car certains arrêts de la Cour de Justice préparent les réalisations des commissions Delors (arrêt Cassis de Dijon de 1979 sur la reconnaissance mutuelle, par exemple).

Si aucun consensus n'émerge sur l'histoire de l'intégration européenne, signe de vitalité intellectuelle de ce champ, force est de constater que le débat reste animé par des universitaires adoptant une méthode historique, plus que par historiens «stricto sensu ». Cette situation s'explique toutefois de manière logique.

\section{Des difficultés spécifiques}

L'histoire de l'intégration européenne souffre d'un certain nombre de handicaps structurels lié à l'objet d'étude même. La diversité linguistique et culturelle est un premier obstacle. Certes, aucune étude historique sérieuse ne peut être menée aujourd'hui sans prendre en compte les historiographies étrangères, mais cette exigence concerne aussi les sources primaires pour la nouvelle histoire de l'intégration européenne, fondée sur une démarche multi-archives. Plus généralement, les difficultés propres à l'histoire comparative s'imposent, comme le fait que des concepts apparemment voisins n'ont pas le même sens dans chaque pays. Pierre Bourdieu a souligné les « facteurs structuraux qui sont générateurs de malentendus » comme « le fait que les textes circulent sans leur contexte », ou que les références étrangères soient utilisées en fonction des contextes de réception (Bourdieu, 2002, 4). 
Ainsi la notion d' « exploitation familiale » qui est au cœur de la PAC n'a pas la même signification dans tous les pays européens. La même remarque vaut pour les «crises » et les « relances » dont les chronologies varient en fonction des pays en fonction de leur date d'adhésion à la CEE, mais aussi de nuances internes. Ainsi l'historiographie allemande souligne-t-elle le rôle de la présidence allemande de 1983 dans la « relance » du processus d'intégration européenne (Stark, 2004, 34-36), alors que les chercheurs français insistent sur le rôle du sommet de Fontainebleau de 1984 sous présidence française (Saunier, 2011, 22).

Par ailleurs, la recherche multi-archives souffre d'un certain nombre de contraintes pratiques. La diversité des fonds implique un manque de correspondance entre eux. Ainsi, une situation perçue comme une crise grave dans un pays donné fera l'objet de nombreux développements, jalousement conservés dans les archives, alors qu'elle sera considérée comme un phénomène mineur et largement ignoré dans un autre pays. La relativité de la notion de crise est ici exemplifiée. D’autres crises sont simplement ignorées par les lacunes physiques des archives. Les fonds de l'OCDE sont souvent très superficiels par exemple, ce qui s'explique par les pouvoirs faibles dévolus à cette institution. Au sein de l'UE, les fonds sont parfois moins explicites que les archives nationales car la tradition de collecte est plus récente, cependant que l'accès demeure toujours problématique pour les archives de la Cour de justice.

De plus, comme toute histoire du temps présent, l'histoire de l'intégration européenne est sensible à la demande institutionnelle publique, en particulier des institutions de l’Union européenne. S’est ainsi développée une histoire officielle qui contribue à perpétuer une approche traditionnelle des institutions européennes (Mangenot et Schirmann, 2012). À titre d'illustration, l'Histoire de la Commission européenne 1958-1973 (Dumoulin, 2007) est très riche et d'une grande qualité technique, mais est dépourvue de toute démarche interprétative (Ludlow, 2010, 118). Elle repose sur une approche d'histoire administrative interne qui entretient le cliché d'une histoire de l'intégration européenne narrative, descriptive et autocentrée, largement fondée sur la vision classique du cycle crise/relance. L'histoire officielle de la Commission européenne est néanmoins intéressante pour ses apports empiriques, et pour les sources qu'elle offre aux chercheurs. Elle contribue en effet à l'ouverture des archives et repose sur une vaste campagne d'archives orales librement accessibles car toutes les interviews, et leur transcription écrite, sont librement disponibles. Le souci de transparence contraste avec les entretiens privés 
utilisés par la plupart des autres chercheurs. De nouvelles interprétations peuvent alors être proposées sur la base de ce premier matériau.

Sur le plan épistémologique, la perception d'une crise de l'histoire de l'intégration européenne est amplifiée par le défi posé par la science politique. La critique de travaux d'historiens trop narratifs et trop descriptifs provient de cette comparaison car toutes les approches historiques sont menacées par ces travers. L'interpénétration entre les deux disciplines devrait d'ailleurs s'accroître à l'avenir, lorsque les archives de la période Delors seront disponibles et exploitées par les historiens, car ces années sont déjà très largement étudiées par les politistes. Le dialogue avec la science politique est toutefois un défi ancien pour les historiens, comme le montre l'exemple de Pierre Gerbet, historien devenu politiste (Dulphy et Manigand, 2007). Dès 1975, Alan Milward appelait à une coopération accrue entre historiens et représentants des autres sciences sociales (Milward, 1975, 73-75). En 1995, il encourageait même les historiens à forger leurs propres concepts et théories interprétatives, en particulier autour de la notion d'« allégeance » à une autorité, c'est-à-dire la loyauté envers une institution (nationale et/ou supranationale) (Milward, 1995). Il s'éloignait ainsi de la vision classique en termes de crise et de relance, en proposant une grille d'analyse plus transversale. Cependant, il n'a pas pu mettre en œuvre son programme du fait de sa concentration sur la thématique plus classique de la politique européenne de la Grande-Bretagne et des élargissements (Milward, 2002, 2005). Wolfram Kaiser a réfléchi plus précisément aux coopérations possibles entre politistes et historiens qui dépasseraient les clichés éculés, les premiers considérant les seconds comme de sympathiques tâcherons incapables de conceptualiser, et les seconds se méfiant des généralisations hâtives et superficielles des premiers. Pour Kaiser, l'utilisation de concepts de sciences politiques par les historiens leur permettrait d'être moins descriptifs et de proposer une hiérarchie entre les facteurs explicatifs (Kaiser, 2010, 63; Kaiser, 2008, 309). Il applique d'ailleurs cette démarche aux réseaux transnationaux depuis maintenant une petite dizaine d'année, preuve du renouvellement des méthodes (Kaiser et Starie, 2005). Finalement le « challenge interdisciplinaire » (Warlouzet, 2014) commence à être relevé par certains historiens, préparant la voie à une véritable « relance » de la discipline. 


\section{La relance de l'histoire de l'intégration européenne}

Le constat de crise de l'histoire de l'intégration européenne apparaît largement exagéré après une analyse approfondie, ce qui permet de dresser les voies de sa relance par «l'histoire des coopérations européennes».

\section{Une critique caricaturale}

Le topos de la critique d'une histoire considérée comme autocentrée et téléologique est désormais largement éculé, sauf si l'on arrête le curseur en 1990. C'est ce qui peut ressortir d'une lecture rapide de l'article de Mark Gilbert de 2008 (Gilbert, 2008), qui ne se veut d'ailleurs pas un état des lieux exhaustif de l'historiographie. En effet, l'auteur concentre ses critiques sur Bino Olivi, qui n'est ni historien ni même universitaire, et sur des historiens de la génération des pionniers, en l'occurrence Walter Lipgens (1925-1984), Jean-Baptiste Duroselle (1917-1994) et Pierre Gerbet (1918-2009). Les autres historiens cités sont, en dehors d'Alan Milward, plus jeunes (Elvert, Gillingham, Ludlow) mais ils ne sont pas directement critiqués. Par la suite, M. Gilbert a complété son analyse en distinguant explicitement les publications évitant l'écueil de la téléologie, et celles qui y ont toujours recours. Il a le mérite de cibler notamment une contribution caricaturale de l'historien allemand Wilfried Loth opposant le vertueux Walter Hallstein au rétrograde Charles de Gaulle pendant la crise de la Chaise vide (Gilbert; 2010, 176177). Or les conceptions européennes des deux hommes ont produit des effets plus complexes qu'il n'y parait. De Gaulle a même pu passer pour un « Père de l'Europe » pour son rôle dans le succès initial, et improbable, de la CEE (Warlouzet, 2011b). De fait, l'histoire de l'intégration européenne, et en particulier de ses crises, a évolué depuis le fédéraliste Walter Lipgens. Sans prétendre à l'exhaustivité, une série de courants peut être identifiée.

La critique d'une histoire autocentrée a été largement dépassée par les études alliant l'histoire des relations internationales et l'histoire socio-économique et pratiquées notamment à l'Université de Padoue autour d'Antonio Varsori depuis une quinzaine d'années. Cette école padouane réinsère l'histoire de la CECA-CEE dans une histoire globale des organisations internationale (OITBureau international du travai [BIT], Organisation des Nations unies [ONU], OCDE avec Lorenzo Mechi ou Francesco Petrini) ou de la guerre froide (David Burigana et Angela Romano). Répondant au développement de l'histoire globale, un accent particulier a été mis sur l'articulation entre l'intégration 
européenne et les coopérations Nord/Sud par le biais de la Conférence des Nations unies sur le commerce et le développement (CNUCED) ou d'accords de coopération entre la CEE et les pays associés, situés essentiellement en Afrique à l'origine (accord de Yaoundé, 1963) puis répartis de manière plus large ensuite (Garavini, 2012 ; Migani, 2008). Cette approche est maintenant adoptée par de nombreux chercheurs, comme en témoigne un colloque récent sur l'interaction entre les sommets européens et mondiaux dans les années 1970 et 1980 (Mourlon-Druol et Romero, 2013).

Plus généralement, la critique d'une histoire limitée au trio CECA-CEE-UE est devenue inexacte depuis longtemps. En 1988, Gérard Bossuat soutenait une thèse de doctorat qui reliait les réflexions de la fin de la Deuxième Guerre mondiale aux débuts du processus de construction européenne en évoquant non seulement la CECA, mais aussi en étudiant également longuement la création de l'OECE. Elle a été publiée en 1992 (Bossuat, 1992). Depuis, de nombreux travaux se sont intéressés à l'avant 1950, en particulier à propos des interactions entre le débat sur l'organisation de l'Europe et celui concernant la Société des Nations (SDN) (Guieu, 2008). Une partie de la foisonnante historiographie de la SDN peut d'ailleurs être rattachée à l'histoire de l'intégration européenne tant les Européens jouèrent un rôle prépondérant dans cette institution (Bussière, 2005). De ce point de vue, les premières difficultés de l'intégration européenne datent de la crise de la sécurité collective dans les années 1930. Les ambitieux projets des années 1920, notamment le plan Briand de 1929, sont alors définitivement enterrés. Il faut ainsi considérer la Première Guerre mondiale comme la crise fondatrice de l'idée européenne sous sa forme contemporaine.

La dénonciation d'une histoire strictement diplomatique et enfermée dans le paradigme de l'État-monolithique est tout aussi caricaturale. Les travaux renouvelant l'approche diplomatique par une prise en compte des divisions internes aux États et aux institutions communautaires ont déjà été cités, notamment autour de Piers Ludlow ou d'Ann-Christina Knudsen. Reste à évoquer le cas des acteurs non-étatiques, qui ont fait l'objet de recherches très nombreuses. L'historiographie francophone s'est distinguée dans l'étude des milieux économiques (Badel, 2011; Bussière, Dumoulin et Schirmann, 2010), tandis que Wolfram Kaiser a lancé un large chantier sur les réseaux transnationaux initiés par des démocrates-chrétiens (Kaiser, 2007). L’approche par les acteurs non-étatiques et les réseaux permet de mettre en valeur des permanences de long terme, au-delà des crises diplomatiques. Ce sont alors moins les crises que les dynamiques de fond expliquant les 
relances qui sont mises en valeur, par exemple autour du rôle des réseaux démocrates-chrétiens dans les initiatives des années 1950 (CECA, CED, CEE), ou des milieux économiques dans le programme du marché unique de 1985. Par ailleurs, les problématiques socioculturelles ont largement irrigué le champ. Les recherches sur l'identité européenne conduites depuis les années 1990 sont maintenant devenues des classiques (Frank, 2004). Elles permettent de mieux comprendre les ressorts profonds des crises, dont le déclenchement diplomatique n'est parfois que la traduction de divergences culturelles importantes. La crise de légitimité dont souffrent les institutions européennes est également étudiée, soit dans une optique nationale (Dulphy et Manigand, 2006), soit à travers le débat sur l'existence d'un « espace public européen » (Frank et al., 2010). Ce rapide survol de la diversité foisonnante de la production des deux dernières décennies permet de considérer que la crise de la discipline est un phénomène révolu, et correspond à la situation du tournant des années 1990.

\section{Vers une relance de l'histoire de l'intégration européenne}

Pour leurs promoteurs, la relance de l'histoire de l'intégration européenne passe d'abord par l'affirmation de l'intérêt objectif considérable de ce champ d'étude. Si ce constat paraît évident pour les juristes ou les politologues, qui tous bénéficient de nombreux postes, voire de départements dédiés à ce domaine, il reste à convaincre certains historiens. La crise de légitimité de l'Union européenne a débuté avec sa naissance, en 1992. C'est en effet à partir de cette date que le « consensus permissif » a laissé place au « déficit démocratique ». Le phénomène est ensuite allé en s'amplifiant, et a été renforcé par une contestation plus générale de l'UE, qui déboucha sur les deux référendums de 2005 en France et au Pays Bas rejetant le traité constitutionnel. Cette crise de l'Union européenne a sans doute été un élément majeur dans la perception d'une crise de l'histoire de cette organisation, dans la mesure où la construction d'un récit national (ou ici supranational) partagé passe par un travail de construction historique. Les historiens étaient alors accusés de participer au façonnage d'une identité artificielle et/ou élitiste dans une approche téléologique. Cette critique est largement exagérée, comme en témoignent les divisions importantes existantes entre historiens. Ainsi, deux travaux sur archives récents portant sur la naissance de la politique européenne de la concurrence défendent des conclusions opposées quant au rôle relatif des États d'une part, et de la Commission européenne d'autre 
part (Hambloch, 2009; Pitzer, 2009). Aucun unanimisme ne règne donc sur l'interprétation du processus d'intégration européenne.

D’une manière générale, il est certain que tout historien est tenté par le réflexe téléologique, dans la mesure où il connaît le présent et où il a une certaine empathie avec le sujet. Le risque existe aussi dans l'histoire transnationale, généralement identifiée comme la nouvelle frontière de l'histoire de l'intégration européenne. Les historiens du transnational sont conscients du risque de cette dérive, rappelé par Patricia Clavin dans un article programmatique où elle dénonçait « a tendency in the literature to date to present transnationalist encounters as consistently progressive and co-operative in character» portant le risque d'une « teleological history of globalisation » (Clavin, 2005, 424). De même, Sandrine Kott souligne que la nécessité d'étudier les dynamiques transnationales et internationales «n'invalide pas le rôle joué par les États, pas plus qu'elle n’entame la prégnance du référentiel national » (Kott, 2011 , 8). Comme tous les historiens, ceux s’intéressant à la construction européenne doivent donc s'efforcer de garder une distance critique envers tous les acteurs, et, sans perspective moralisatrice, souligner les failles de chacun. Il est notamment nécessaire de garder en mémoire les alternatives non réalisées afin de souligner l'importance de la contingence.

Bien plus, même si l'Union européenne s'écroulait demain, elle resterait un phénomène historique considérable. C'est le seul phénomène connu d'un transfert massif, volontaire et pacifique de pouvoirs d'États à des institutions communautaires dont la logique est en partie fédérale. De multiples organisations régionales ont été créées de par le monde mais elles conservent toutes une dimension très largement intergouvernementale. Ce phénomène peut être certes être critiqué, mais ne peut être tenu pour mineur, comme une simple conséquence de la Guerre froide par exemple. La comparaison des relations franco-allemandes d'une part, marquée par une réconciliation durable et profonde, et celles existantes entre le Japon et la Corée du Sud d'autre part, toujours marquée par une hostilité réciproque, permet de prendre conscience de l'influence spécifique de l'intégration européenne. Cette différence survient alors même que tous ces pays (France, RFA, Japon, Corée du Sud) appartenaient au même camp pendant la Guerre froide. Enfin, la crise de l'Euro elle-même montre combien les études économiques et institutionnelles centrées sur l'Union européenne sont fondamentales pour répondre à la demande sociétale. L'intérêt heuristique du domaine reste donc considérable, comme le prouve la conversion à ce champ d'étude d'historiens 
dont les recherches précédentes concernaient d'autres domaines (Bernard Bruneteau, Michel Catala et Kiran Patel).

L'enjeu majeur reste de mettre en valeur les recherches foisonnantes entreprises depuis deux décennies. C'est l'esprit de la question « Penser et construire l'Europe, 1919-1992 » proposée aux concours de recrutement au professorat d'histoire-géographie en 2008-2009 (Guieu et Raflik, 2007). De la crise de la Première Guerre mondiale à la crise post-Maastricht, une unité est donnée par l'ambition européenne, et par une certaine distanciation chronologique qui favorise l'analyse historique.

\section{L’histoire des coopérations européennes}

Reste le problème cardinal de la dénomination du champ, avec le terme d'« intégration ». Associé à l'idée de fusion en un tout, le terme renvoie au premier considérant des traités de Rome: « [Les contractants sont] déterminés à établir les fondements d'une union sans cesse plus étroite entre les peuples européens ». Dès 1992, A. Milward dénonçait le caractère téléologique de ce mot, qui impliquait une fusion progressive des économies, des sociétés et des institutions nationales (Milward, 1992, 3). Cela ne semble d'ailleurs pas déranger certains historiens comme W. Kaiser, pour qui il faut même passer de l'histoire de l'intégration européenne à « l'histoire de l'Europe intégrée » (Kaiser, 2010, 65). Le biais téléologique est ainsi explicitement confirmé. Plus justement, Giuliano Garavini estime qu'il faudrait parler des intégrations européennes au pluriel, afin de prendre en compte la diversité des phénomènes (Garavini, 2012, 5). Toutefois, le terme «intégration », comme celui de « construction », porte en lui une dimension téléologique. Les expressions « Histoire de l'Europe » ou « Histoire des Européens » sont quant à elles trop générales. Il faudrait donc parler d'« histoire des coopérations européennes ». Cette expression désigne l'ensemble des interactions entre États et acteurs non-étatiques européens depuis 1919, en mettant en valeur tout à la fois leur diversité et leur intensité. Elle permettrait ainsi de resituer leur spécificité dans une comparaison globale entre différents types de coopérations régionales, ou avec les coopérations à l'échelle mondiale. Cette dénomination a l'avantage de prendre explicitement en compte tous les projets et réalisations à caractère purement intergouvernemental, sans isoler le trio CECA-CEE-UE qui deviendrait un sous-ensemble de cette histoire. Elle permettrait aussi de mieux comprendre la diversité interne de l'Union européenne, où des domaines très fédéralisés (politique de la concurrence, 
politique monétaire) voisinent avec des politiques intergouvernementales (défense, diplomatie). Enfin, elle permettrait de mieux envisager l'histoire des mondialisations, en articulant les dimensions européennes, inter-régionales, et globales. La dénomination « histoire des coopérations européennes » rendrait ainsi justice au profond renouvellement de l'histoire de l'intégration européenne lors des deux dernières décennies.

\section{Conclusion}

La spécificité de la notion de « crise » dans l'histoire de l'intégration européenne tient à son importance à la fois dans les discussions historiques et dans les bilans historiographiques décrivant la situation de la discipline. Il semble que la crise de légitimité affectant l'Union européenne depuis les années 1990 affecte la recherche historique sur cet objet. Le savant et son objet se rejoignent alors dans une dialectique crise relance de long terme. Cette dynamique commence avec le discours Schuman de 1950 dans l'historiographie classique, largement critiquée pour son caractère autocentré et sa tentation téléologique.

Ce constat de crise de l'histoire de l'intégration européenne est toutefois largement caricatural et doit être dépassé. Le champ s'est largement renouvelé tant dans ses méthodes que dans ses sujets. Le recours à une méthode de recherche multi-archives et à la prise en compte d'acteurs de plus en plus diversifiés permet de sortir de l'histoire traditionnelle, fédéraliste ou purement intergouvernementale, pour aboutir à une compréhension plus fine des interactions entre les différentes échelles, en particulier nationales et européennes. La perspective chronologique s'élargit maintenant à l'ensemble du court $\mathrm{xx}^{\mathrm{e}}$ siècle (1914-1989) en remontant à la Première Guerre mondiale. De nouvelles perspectives sont adoptées comme le constructivisme, le rôle du droit ou l'approche par les réseaux transnationaux. Le prisme crise/ alternance n'est pas discrédité pour autant mais il s'est complexifié. Ainsi, l'épisode de la « Chaise de vide » de 1965-1966 reste une crise, mais son interprétation a été considérablement complexifiée pour sortir de l'histoire héroïsante des débuts, nourris des affrontements entre « grands hommes ».

La notion de « crise » n'est pas forcément négative car elle peut aussi amorcer un renouveau. L'histoire de l'intégration européenne est aujourd'hui sortie de cette crise, même si elle connaît toujours un problème d'identité. Le 
recours à la notion d' " histoire des coopérations européennes » permettrait de mieux identifier un domaine d'étude aux potentiels considérables, et de dépasser l'ambiguïté téléologique du terme « intégration ». Enfin, le retour sur l'histoire permettrait de mieux appréhender les crises actuelles, mais aussi de mesurer la portée des tentatives de relance, au-delà de la succession des Conseils européens présentés comme « décisifs » pour régler la crise.

\section{Références bibliographiques}

Akman Pinar (2009), "Searching for the Long-Lost Soul of Article 82EC », Oxford Journal of Legal Studies, vol. 29, $\mathrm{n}^{\circ}$ 2, p. 267-303.

Aron Raymond et Lerner Daniel (dir.) (1956), La querelle de la CED, Paris, Armand Colin.

Badel Laurence et Michel Hélène (2011), « Introduction », in Laurence Badel et Hélène Michel (dir.), Patronats et intégration européenne. Pour un dialogue disciplinaire raisonné, Paris, L’Harmattan, p. 9-16.

Badel Laurence (2011), « Organisation patronales et intégration européenne au prisme de l'école historique française des Relations Internationales », in Laurence Badel et Hélène Michel (dir.), Patronats et intégration européenne. Pour un dialogue disciplinaire raisonné, Paris, L’Harmattan, p. 133-154.

Bourdieu Pierre (2002), « Les conditions sociales de la circulation internationale des idées ", Actes de la recherche en sciences sociales, 2002/5, $\mathrm{n}^{\circ} 145$, p. 3-8.

Bussière Éric (2005), « Premiers schémas européens et économie internationale durant l'entre-deux-guerres », Relations Internationales, $\mathrm{n}^{\circ} 123$, p. $51-70$

Bussière Éric, Dumoulin Michel, et Schirmann Sylvain (2010), « Le développement de l'intégration économique », in Gérard Bossuat, Éric Bussière, Michel Dumoulin et al. (dir.), L'expérience européenne. 50 ans de construction européenne, Bruxelles, Bruylant, p. 56-137.

Clavin Patricia (2005), « Defining Transnationalism », Contemporary European History, vol. 14, n 4, p. 421-439. 
Davies Billy et Rasmussen Morten (dir.) (2012), « Towards a New History of Europea Law », special issue of Contemporary European History, vol. 21, $\mathrm{n}^{\circ} 3$.

Deighton Anne (1990), « Missing the Boat: Britain and Europe, 1945-1961 », Contemporary Record, vol. 3, $\mathrm{n}^{\circ}$ 3, p. 15-17.

Cohen Antonin (2007), « Le "père de l'Europe”. la construction sociale d'un récit des origines ", Actes de la recherche en sciences sociales, $\mathrm{n}^{\circ}$ 166-167, p. 14-29.

Dulphy Anne et Manigand Christine (2006), La France au risque de l'Europe, Paris, Armand Colin.

Dulphy Anne et Manigand Christine (2007), « Entretien avec Pierre Gerbet, professeur émérite à l'Institut d'études politiques de Paris », Histoire@ Politique, $\mathrm{n}^{\circ}$ 02, disponible sur: <http://www.histoire-politique.fr>.

Dumoulin Michel (dir.) (2007), La Commission européenne, 1958-1972. Histoire et mémoire d'une institution, Bruxelles, Communautés européennes.

Frank Robert (dir.) (2004), Les identités européennes au $x x^{e}$ siècle. Diversités, convergences et solidarités, Paris, Publications de la Sorbonne.

Frank Robert, Kaelble Hartmut, Lévy Marie-Françoise et al. (2010), « La construction d'un espace public européen ", in Gérard Bossuat, Éric Bussière, Michel Dumoulin et al. (dir.), L'expérience européenne. 50 ans de construction européenne, Bruxelles, Bruylant, p. 171-234.

Garavini Giuliano (2012), European Integration, Decolonization, and the Challenge from the Global South, 1957-1986, Oxford, Oxford University Press.

Gilbert Mark (2008), « Narrating the Process: Questioning the Progressive Story of European Integration », Journal of Common Market Studies, vol. $46, \mathrm{n}^{\circ} 3$, p. 641-662.

Gilbert Mark (2010), « A Polity Constructed: New Explorations in European Integration History », Contemporary European Integration History, vol. 19, $\mathrm{n}^{\circ} 2$, p. 169-179.

Gillingham John (2003), European Integration, 1950-2003. Superstate or New Market Economy?, Cambridge, Cambridge University Press.

Guieu Jean-Michel (2008), Le rameau et le glaive. Les militants français pour la Société des Nations, Paris, Les Presses de Sciences Po. 
Guieu Jean-Michel et Raflik Jenny (2007), « Le point sur : Penser et construire l’Europe, 1919-1992 », Historiens \& Géographes, $n^{\circ} 399$, p. 145-183.

Guieu Jean-Michel et al. (2007), Penser et construire l'Europe, 1919-1992, Paris, Belin.

Hambloch Sybille (2009), Europaïsche Integration und Wettbewerbspolitik. Die Frühphase der EWG, Baden-Baden, Nomos.

Harst Jan van der (2007), Beyond the Custom's Union: the European Community's Quest for Deepening, Widening and Completion, 1969-1975, Bruxelles, Bruylant.

Kaiser Wolfram (1996), Using Europe, Abusing the Europeans. Britain and European Integration, 1945-1963, Basingstoke, Macmillan Press.

Kaiser Wolfram (2001), « Culturally Embedded and Path-dependent: Peripheral Alternatives to ECSC/EEC “Core” Europe », Revue d'histoire de l'intégration européenne, vol. $7, \mathrm{n}^{\circ}$ 2, p. 11-36

Kaiser Wolfram (2005), « From State to Society? The Historiography of European Integration ", in Michelle Cini et Angela Bourne (dir.), Palgrave Advances in European Union Studies, Chippenham, Plagrave Macmillan, p. 190-208

Kaiser Wolfram (2007), Christian Democracy and the Origines of the EU, Cambridge, Cambridge University Press.

Kaiser Wolfram (2008), « History meets Politics: Overcoming Interdisciplinary Volapük in Research on the EU », Journal of European Public Policy, vol. 15, $\mathrm{n}^{\circ} 2$, p. 300-313.

Kaiser Wolfram (2010), « From Isolation to Centrality: Contemporary History Meets European Studies ", in Wolfram Kaiser et Antonio Varsori (dir.), European Union History. Themes and Debates, Chippenham, Palgave Macmillan, p. 45-65.

Kaiser Wolfram et Starie Peter (dir.) (2005), Transnational European Union. Towards a Common Political Space, Londres, Routledge.

Kott Sandrine (2011), « Les organisations internationales, terrains d'étude de la globalisation. Jalons pour une approche socio-historique », Critique internationale, $\mathrm{n}^{\circ}$ 52, p. 9-16.

Knudsen Ann-Christina (2009), Farmers on Welfare. The Making of Europe's Common Agricultural Policy, Ithaca et Londres, Cornell University Press. 
Lieshout Robert H., Segers Mathieu L. et van der Vleuten Anna (2004), « De Gaulle, Moravcsik, and The Choice for Europe, Soft Sources, Weak Evidence », Journal of Cold War Studies, vol. 4, nº 6, p. 89-139.

Lindseth Peter (2010), Power and Legitimacy. Reconciling Europe and the Nation-State, Oxford, Oxford University Press.

Ludlow N. Piers (1997), Dealing with Britain. The Six and the first UK application to the EEC, Cambridge, Cambridge University Press.

Ludlow N. Piers (2006), The European Community and the Crises of the 1960s. Negotiating the Gaullist challenge, Londres, Routledge.

Ludlow N. Piers (2007), « La politique européenne de Thatcher (1979-1990). L'itinéraire d'un libéralisateur frustré? », in Éric Bussière, Michel Dumoulin et Sylvain Schirmann (dir.), Milieux économiques et intégration européenne au $x^{e}$ siècle. La relance des années quatre-vingt (1979-1992), Paris, Cheff, p. 165-181.

Ludlow N. Piers (2010), « Governing Europe: Charting the Development of a Supranational Policial System », in Wolfram Kaiser et Antonio Varsori (dir.), European Union History. Themes and Debates, Chippenham, Palgave Macmillan, p. 118.

Mangenot Michel et Schirmann Sylvain (2012) (dir.), Les institutions européennes font leur histoire: regards croisés soixante ans après le traité de Paris, Bruxelles, Peter Lang.

Marjolin Robert (1986), Le travail d’une vie. Mémoires (1911-1986), Paris, Robert Laffont.

Mechi Lorenzo (2012), L'organizzazione Internazionale del Lavoro e la ricostruzione europea. Le basi sociali dell'integrazione economica (1931-1957), Roma, Ediesse.

Mechi Lorenzo (2006), « Les États membres, les institutions et les débuts du Fond Social Européen ", in Antonio Varsori (dir.), Inside the European Community. Actors and Policies in the European Integration 1957-1972, Bruylant, Bruxelles, p. 95-116.

Mégie Antoine et Sacriste Guillaume (2009), " Polilexes : champ juridique européen et polity communautaire », rubrique "Chantier de recherche », Politique européenne, $\mathrm{n}^{\circ}$ 28, $\mathrm{p}$ 157-162. 
Migani Guia (2008), La France et l'Afrique Sub-saharienne, 1957-1963. Histoire d'une décolonisation entre idéaux eurafricains et politique de puissance, Bruxelles, Peter Lang.

Milward Alan S. (1975), "The European Studies Movement: What's in a Name? Milward », Journal of Common Market Studies, vol. 14, n 1, p. 69-80.

Milward Alan S. (1992) (with the assistance of George Brennon and Frederico Romero), The European Rescue of the Nation-State, Londres, Routledge.

Milward Alan S. (1995), « Allegiance. The Past and the future », Revue d'histoire de l'intégration européenne, vol. 1, $\mathrm{n}^{\circ}$ 1, p. 7-19.

Milward Alan S. (2002), The Rise and Fall of a National Strategy, 1945-1963, Londres, Whitehall History Publishing.

Milward Alan S. (2005), Politics and Economics in the History of the European Union, Londres, Routledge.

Moravcsik Andrew (1998), The Choice for Europe. Social Purpose and State Power from Messina to Maastricht, Ithaca, Cornell University Press.

Mourlon-Druol Emmanuel (2012), A Europe made of Money. The Emergence of the European Monetary System, Ithaca et Londres, Cornell University Press.

Mourlon-Druol Emmanuel et Romero Federico (2013), International Summitry and Global Governance: the Rise of the G7 and the European Council, 19741991, Londres, Routledge.

Olivi Bino et Giacone Alessandro (2007), L'Europe difficile. La construction européenne, Paris, Gallimard.

Parsons Craig (2003), A Certain Idea of Europe, Ithaca, Cornell University Press.

Patel Kiran Klaus (2009), « Europeanisation à contre coeur. West Germany and agricultural Integration, 1950-1975 », in Klaus Kiran Patel (dir.), Fertile Ground for Europe? The History of European Integration and the Common Agricultural Policy since 1945, Baden-Baden, Nomos Verlag, p. 139-160.

Patel Kiran Klaus (2013), «Provincialising European Union: Co-operation and Integration in Europe in a Historical Perspective », Contemporary European History, vol. 22, no 4, p. 649-673. 
Pitzer Frank (2009), Interessen im Wettbewerb. Grundlagen und frühe Entwicklung der europaïschen Wettbewerbspolitik, 1955-1966, Stuttgart, Franz Steiner.

Rittberger Berthold (2005), Building Europe's Parliament: Democratic Representation beyond the Nation-State, Oxford, Oxford University Press.

Saunier Georges (2011), « De la Communauté à l'Union européenne. L'action européenne de François Mitterrand », Matériaux pour l'histoire de notre temps, $\mathrm{n}^{\mathrm{o}} 1$, p. 20-28.

Stark Hans (2004), Kohl, l'Allemagne et l'Europe. La politique d'intégration européenne de la République fédérale, 1982-1998, Paris, L’Harmattan.

Vauchez Antoine (2013), L'Union par le droit: l'invention d'un programme institutionnel pour l'Europe, Paris, Presses de Sciences Po.

Warlouzet Laurent (2011a), Le choix de la CEE par la France. Les débats économiques de Pierre Mendès-France à Charles de Gaulle (1955-1969), Paris, Cheff.

Warlouzet Laurent (2011b), « De Gaulle as a Father of Europe: The Unpredictability of the FTA's Failure and the EEC's Success (1956-1958) », Contemporary European History, vol. 20, n 4, p. 419-434.

Warlouzet Laurent (2014, à paraître), « The Interdisciplinary Challenge in European Integration History », Journal of Contemporary History.

\section{Laurent Warlouzet}

Marie Curie Fellow, London School of Economics and Political Science I.d.warlouzet@|se.ac.uk 